\title{
Quantitative Approximability of Optimal Control by Linear Programing Model for Asymptomatic Dual HIV - Pathogen Infections
}

\author{
Bassey, E. Bassey ${ }^{*}$ \\ *Department of Mathematics /Statistics, Cross River University of Technology, Calabar, Nigeria \\ *Corresponding Author: Bassey, E. Bassey, Department of Mathematics /Statistics, Cross River \\ University of Technology, Calabar, Nigeria
}

\begin{abstract}
Propelled by the weakness of some notable scientific investigations and the continual desire to achieve a more précised result for the eradication of dual HIV-pathogen infections, the present paper formulated a 7-Dimensional nonlinear mathematical dynamic model presupposed to account for the optimal treatment of asymptomatic dual HIV-pathogen infections studied under quadrupled treatment functions. The model was presented as a linear optimal control time problem (LOCTP) analyzed using linear programing approximability approach, embedded with theoretical measured space. The study as well established the positivity and boundedness of model state variables and numerical simulations conducted. Results of simulations clearly raises positive variations in comparison of healthy $\mathrm{CD}^{+} \mathrm{T}$ lymphocytes count, dual HIVpathogen infections, critical role of dual CTLs and the aggressiveness of virions before and after the introduction of multiple chemotherapy treatment. Therefore, in justifying the application of linear programing approximation model, the study strongly advocates the articulation of delay intracellular into the state variables for enhancement of future investigation.
\end{abstract}

Keywords: quantitative-approximability, linear-programing, asymptomatic, quadrupled, functional-space, measured-space, Lebesgue-measurable

\section{INTRODUCTION}

It has become obvious that the human race have been skewed to the realization of the painful fact that human immunodeficiency virus (HIV) in their negative affinity, have become an important integral component of the human immune systems with the later as primary victim. This negativity in affiliation has rather place the scientific community and beyond in a most seeming precarious dehumanization following the near insurmountable and colossal activities of the deadly disease, which have been without outright medical cure. A situation, which often transmute to full-blown acquired immunodeficiency syndrome (AIDS) with lethal outcome as final consequences.

Non-the-less, considerable height has been attained by research scientists towards the prolongation of lifespan of infected patients and in most cases, aimed at the eradication of the dreaded HIV/AIDS disease, see for example [1-7]. Of interest, these notable achievements have been fronted by the central role of mathematical modeling. However, mathematical modeling as a seeming panacea to the curative approach of HIV/AIDS infection are usually confronted with the difficulty of formulating simplified model with précised and coincides state variables and corresponding parameter functions [8-10]. A situation that has multifaceted dimensions and which are better handle by the judgmental abilities of professionals with the objectives of defining possible solutions to an epidemic.

Modeling of epidemiological outbreak are usually determined by a set of compactible state variables, which in the case of HIV/AIDS, we are commonly concern with the maximization of the host target cells in the class of $\mathrm{CD}^{+}$T-lymphocytes, macrophages and follicular dendritic cells; suppression/elimination of the vectors - viral load and parasitoid-pathogen; maximization of natural anti-HIV (adaptive immune effectors response) known as cytotoxic lymphocytes (CTLs). Cytotoxic lymphocytes are often subdivided into precursors (CTLp) and effectors (CTLe). Other considered state components includes: critical role of time-delay immunity period (or delay intracellular) and the 
functioning capacities of therapeutic chemotherapies, which are generally classified into two families of HAART - reverse transcriptase inhibitors (RTI) and protease inhibitors (PIs) [11-16]. In most recent dimensions, indepth evaluations of viral load have resulted to the inclusion of viral aggressiveness index denoted as $r$-state variable. This component describes the intrinsic virulence and thus, has been accorded important stay in determining epidemiological state variables in HIV dynamic models [17-19].

In this present study, references are deduced from related and compactible models with which the scope of this investigation lies. In our attempt to analyze an all-inclusive model that sufficiently represent the biological interplay of acceptable key components for dual HIV-pathogen infections; we invoke a number of closely related models. For instance, model [17] had studied a set of mathematical model of HIV pathogenesis and treatment. The model not only established the usefulness of the application of mathematical model in the dynamics of HIV transmission but as well, affirmed the capability of linear programing model in understanding the correlations of long-term immunological control of HIV. The model [19] motivated by that of [17] had formulated an optimal control model, which accounted for single drug treatment factor and crucial role of immune response (recognized as effector and memory - CTLe/p) for the control of cellular infection rate. The task of model [18] was the maximization of symptomatic stage of fast progressive HIV infected patient using embedding method. The result of this investigation was in affirmation of the technique adopted.

Resourcefully, a critical review of the aforementioned literatures shows that previous investigations have been on single viral load. This situation clearly could not account for the aggressiveness of diverse new cases of HIV and its allies of pathogenic infections. Furthermore, treatment mechanisms for these studies were either single treatment factor or more but never comprehensive. Even the studies [16, 20], where dual HIV infectivity was considered, only single and pair chemotherapies were respectively programmed. Thus, the present study equipped by the identified weakness of the aforementioned literatures, formulates using ordinary differential equations a set of novel 7Dimensional nonlinear mathematical dual HIV dynamic model, principally to account for the optimal control treatment of asymptomatic dual HIV-pathogen infections on target cells $\left(\mathrm{CD}^{+}{ }^{-} \mathrm{T}-\right.$ lymphocytes) under clinical application of quadrupled treatment functions, which includes linear index of virions aggressiveness. The derive model is reformulated as an optimal control problem and analyzed using linear programing approximation method.

The entire investigation is floated as a version of six sections with section 1 covering the introductory aspect. Section 2 defined the material and methods of study, which constitutes the formulation of model mathematical equations and schematic representation of the model. We shall also verify in this section, the non-negativity of model state variables and ensure that existence of solutions is bounded. We devote section 3 to the transformation of derived model to a time optimal control problem with analysis conducted using linear programing approximation method (LP-PM). We present in section 4, related procedures with specified functional and measure spaces. Affirmation of the model and its analysis are explicitly illustrated in section 5 . This section also contains the discussion of the resulting outcome. Finally, in section 6, we draw succinct conclusion and remarks base on investigation. It is hoped that the method adopted here will provide some straightforward approach without the imposition of artificial conditions.

\section{MATERIAL AND METHODS}

We pre-occupy this section with the mathematical formulation of system basic model equations aided with schematic representation. Since the model is a complete representation of living organisms, the section also considers the positivity of model state variables and validity boundedness of solution.

\subsection{Formulation of Mathematical Model}

Drawing from the innovative ideas of section 1, it is of essence that the derivation of the present model takes its offspring from two compactible established models of [18, 20]. From model [18], the interplay of single HIV infection with target cells $-\mathrm{CD}^{+} \mathrm{T}$ cells and natural anti-HIV defense mechanism was investigated. The governing equations for this model were obtained as: 


$$
\begin{aligned}
& \dot{x}=\lambda-d x-r x v \\
& \dot{y}=r x v-\alpha y-\rho y z \\
& \dot{w}=c x y w-q y w-b w \\
& \dot{z}=q y w-h z \\
& \dot{v}=k\left(1-u_{p}\right) y-\tau v \\
& \dot{r}=r_{0}-u_{R}
\end{aligned}
$$

where $x(t), y(t), w(t), z(t), v(t)$ and $r(t)$ denotes the key state components of the model. We refer readers to the cited reference for detail description of used parameter.

An extension of infection dynamics to dual infectivity was considered by model [20], where dual HIV-pathogen model studied using single treatment function - reverse transcriptase inhibitors (RTI) was investigated. The epidemiological optimal control model was derived as:

$$
\begin{aligned}
& \frac{d U_{T}}{d t}=\frac{b}{1+V+P}+g U_{T}\left(1-\frac{U_{T}+I_{T}}{U_{\max }}\right)-\alpha_{1} U_{T}-r(t)\left[h_{1} V U_{T}+h_{2} P U_{T}\right] \\
& \frac{d I_{T}}{d t}=r(t)\left[h_{1} V U_{T}+h_{2} P U_{T}\right]-\left(z_{v}+z_{p}\right) \alpha_{2} I_{T} \\
& \frac{d V}{d t}=z_{v} \alpha_{2} I_{T}-\alpha_{3} V U_{T} \\
& \frac{d P}{d t}=z_{p} \alpha_{2} I_{T}-\alpha_{4} P U_{T}
\end{aligned}
$$

with equation description as contain in cited reference. Thus, for the derivation of standard equations that adequately guarantee the scope of this present study, we utilize the ideas of models (1) and (2) guided by the following assumption.

\section{Assumption 2.1}

i. The dynamics between virions and cytotoxic T-lymphocytes (CTLs) is dependent on host target cells and virions parameters.

ii. Precursors of CTLs exhibit dual characteristic behavior for immune memory replication and effective contamination by virions.

iii. The effective development of CTL memory by precursor of CTL depends on the efficacy and threshold of therapy at initiation point.

iv. High re-establishment of CTL memory is dependent on early initiation of chemotherapy treatment.

Therefore, from the above synopsis, we formulates a novel epidemiological model that accounts for an asymptomatic dual HIV-pathogen infections on host target cells - CD4 $4^{+}$T-lymphocytes under articulated subdivided CTLs and critical role of virulence linear index $R$ with multiple chemotherapies - reverse transcriptase inhibitors (RTI) and protease inhibitors (PIs). So, if the concentration of the present model as characterized by 7-subpopulations is measured in cells per $u l$, then $U_{T}$ uninfected $\mathrm{CD} 4^{+} \mathrm{T}$ cells count, $I_{T}$ - infected $\mathrm{CD} 4^{+} \mathrm{T}$ cells (by both virions), $V$-free viral load, $P$ - free pathogen virus, $W$ - precursor (CTLp), $Z$ - effectors (CTLe) and $R$ - intrinsic virulence index represents the biological state variables. Furthermore, if we let $q_{1}$ and $q_{2}$ denote chemotherapies control functions, then the epidemiological interactions of the components yields the following derive mathematical equations: 


$$
\begin{aligned}
\frac{d U_{T}}{d t} & =\frac{b}{1+V+P}+g U_{T}\left(1-\frac{U_{T}+I_{T}}{U_{\max }}\right)-\alpha_{1} U_{T}-\left(1+q_{1}(t)\right)\left[h_{1} V+h_{2} P\right] U_{T} R \\
\frac{d I_{T}}{d t} & =\left(1-q_{1}(t)\right)\left[h_{1} V+h_{2} P\right] U_{T} R-\left(z_{v}+z_{p}\right) \alpha_{2} I_{T}-\rho I_{T} Z \\
\frac{d V}{d t} & =\left(1-q_{2}(t)\right) z_{v} \alpha_{2} I_{T}-\alpha_{3} V \\
\frac{d P}{d t} & =\left(1-q_{2}(t)\right) z_{p} \alpha_{2} I_{T}-\alpha_{4} P \\
\frac{d W}{d t} & =c U_{T} I_{T} W-\lambda I_{T} W-\alpha_{5} W \\
\frac{d Z}{d t} & =\lambda I_{T} W-\alpha_{6} Z \\
\frac{d R}{d t} & =R_{0}-q_{1}(t) R
\end{aligned}
$$

with initial conditions $U_{T}(0)=U_{(T) 0}, I_{T}(0)=I_{(T) 0}, V(0)=V_{0}, P(0)=P_{0}, W(0)=W_{0}, \mathrm{Z}(0)=Z_{0}$ and $R(0)=R_{0}$ at $t=t_{0}$ and satisfying the biological state variables and parameters as describe in tables $(1 \& 2)$. Thus, model (3) is the standard equation system that satisfies the scope of the present study with biological behavior schematically represented as in fig. 1, below:

Remark 1 It is worth to note that the quadrupled treatment function as applied here includes: reverse transcriptase inhibitors $(R T I)$, protease inhibitors $(P I s)$, cytotoxic T-lymphocytes (CTLs) subdivided into precursors of CTLp and effectors of CTLe.

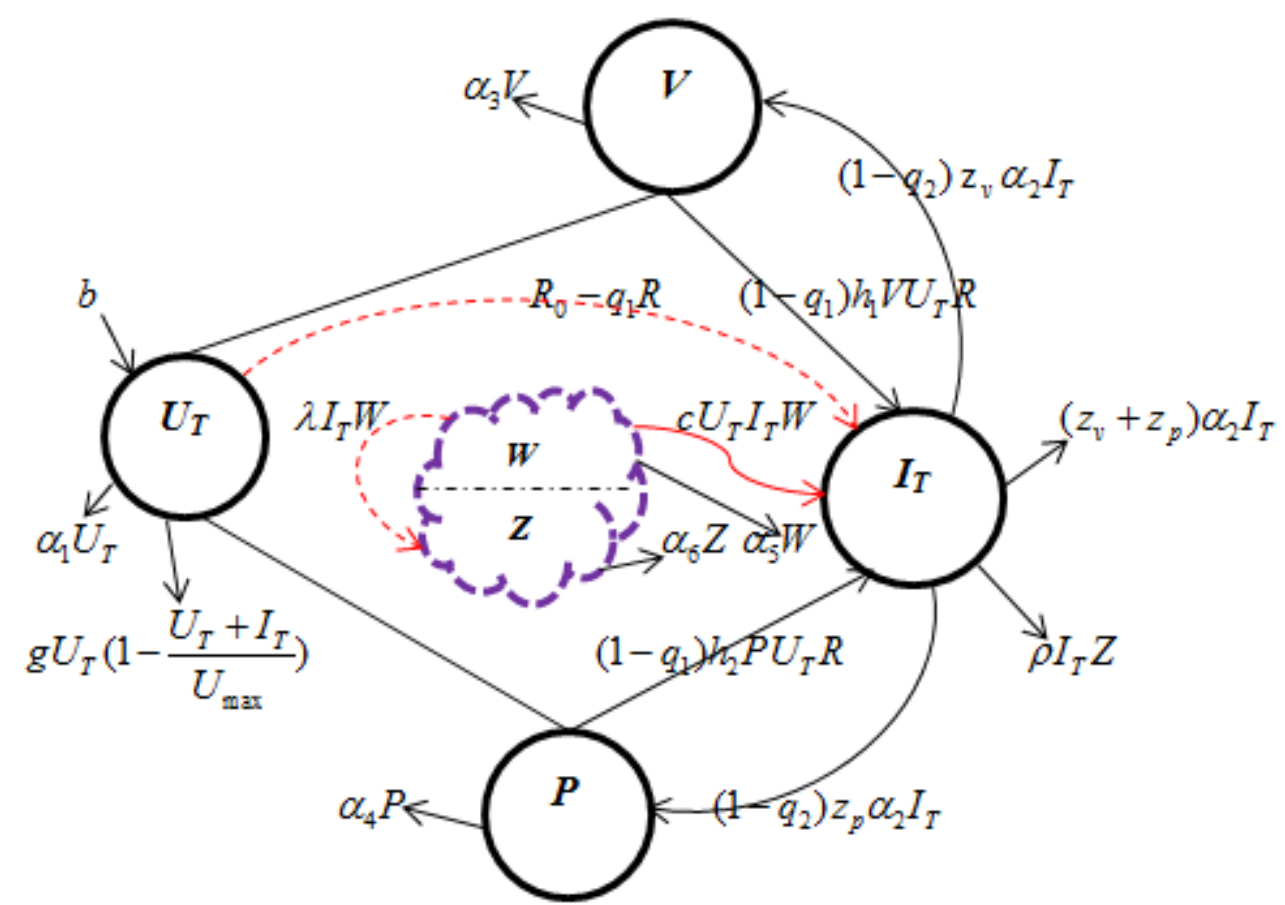

Fig. 1 Schematic representation of dual HIV-pathogen infection with quadruple treatment functions ( RTI, PIs, CTLp, CTLe)

An indepth appreciation of model (3) is informed by the following explicit description of each of terms of the equations. For instance: in the first equation, the first term $\frac{b}{1+V+P}$ denotes natural 
source of uninfected $\mathrm{CD} 4^{+} \mathrm{T}$ cells having a logistic term $g U_{T}\left(1-\frac{U_{T}+I_{T}}{U_{\max }}\right)$. This term account for the fact $U_{T}$ is always never larger than $U_{\text {max }}$. Uninfected cells die natural death at a rate $\alpha_{1} U_{T}$ and is loss due virions at the rates $h_{1} V$ and $h_{2} P$, while $\left(1-q_{1}(t)\right) U_{T} R$ is the infection rate associated with linear index of virions aggressiveness in the presence of control chemotherapy function $q_{1}(t)$.

In the second equation, the first term $\left(1-q_{1}(t)\right)\left[h_{1} V+h_{2} P\right] U_{T} R$ define the inflow of virions infection into susceptible T-cells leading to transmutation to infectious T-cells. The second term $\left(z_{v}+z_{p}\right) \alpha_{2} I_{T}$ describes the clearance rate of infectious cells, which are sustained by virions replication rate $z_{v}$ and $z_{p}$ respectively. The last term $\rho I_{T} Z$ explain the critical role of active immune effectors response in clearing significant amount of infected T-cells.

From third and fourth terms, we define the behavioral tendencies of both virions $V$ and $P$ with inflow of $\left(1-q_{2}(t)\right) z_{v} \alpha_{2} I_{T}$ and $\left(1-q_{2}(t)\right) z_{p} \alpha_{2} I_{T}$ as replicated infectious cells, which are subjected to chemotherapy distortion $q_{2}(t)$. Both equations experience clearance rate of $\alpha_{3} V$ and $\alpha_{4} P$. Taking lead from $[16,20]$, the present model incorporates CTLs population, which is subdivided into precursor of cytotoxic T-lymphocytes - CTLp $(w)$ and effector of cytotoxic T-lymphocytes - CTLe (z). Thus, from fifth equation, the first term $c U_{T} I_{T} W$ denotes the proliferation of CTLp population, which is proportional to both infected T-cells $I_{T}$ and quantified amount of uninfected T-helper cells $U_{T}$. CTLp is differentiated into effectors at the rate $\lambda I_{T} W$, which becomes inflow in the sixth equation. CTLp and CTLe are loss at the rates $\alpha_{5} W$ and $\alpha_{6} Z$.

Finally, the seventh equation describe the dynamics of the intrinsic dual virulence (or aggressiveness of dual virions) denoted by $R$. This index increases linearly for an untreated dual HIV-pathogen infected patient with growth rate that depends on the constant $R_{0}$ and are cleared due to $q_{1}(t)$ at the rate $q_{1}(t) R$.

Remark 2 Precursor of CTLp are responsible for the development of immune memory, while effectors of CTLe are responsible for the active defense of foreign agents i.e. elimination (killing) of virions.

Remark 3 The functions $q_{1}$ and $q_{2}$ are control variables for RTI and PIs with $q_{1}$ acting as active inhibitors that reduces infection rate on healthy $\mathrm{CD}^{+} \mathrm{T}$ cells and growth rate of intrinsic dual virulence. The term $q_{2}$ acts in virions reproduction by inhibiting virions replications from infectious cells.

Remark 4 The constants $h_{1}, h_{2}$ and the $R$-state variable of first, second and seventh equations of present model represents the coefficient $\beta$ of model [17] and state variable of model [21].

From remarks (1and 2), validation of model (3) follows the establishment of realistic values for both state components and parameters as seen in tables 1 and 2 below:

Table 1. Description of model state variables with values

\begin{tabular}{|c|c|c|c|}
\hline \multirow[t]{2}{*}{ Variables } & Dependent variables & \multirow{2}{*}{$\begin{array}{l}\text { Initial } \\
\text { values }\end{array}$} & \multirow[t]{2}{*}{ Units } \\
\hline & Description & & \\
\hline$U_{T}$ & Uninfected T-lymph cells population & 0.4 & cells $\mu l^{-1}$ \\
\hline$I_{T}$ & Infected $\mathrm{CD} 4^{+} \mathrm{T}$-lymphocytes population & 0.2 & cells $\mu l^{-1}$ \\
\hline$V$ & Infectious free viral load population & 0.2 & copiesml $^{-1}$ \\
\hline$P$ & Infectious free pathogen population & 0.1 & copiesml $^{-1}$ \\
\hline
\end{tabular}


Quantitative Approximability of Optimal Control by Linear Programing Model for Asymptomatic Dual HIV - Pathogen Infections

\begin{tabular}{|lll|l|}
\hline$W$ & Precursors of CTLp & 0.02 & ${\text { cells } \mu l^{-1}}^{-1}$ \\
$Z$ & Effectors of CTLe & 0.04 & ${\text { cells } \mu l^{-1}}^{-1}$ \\
$R$ & Intrinsic virulence index & 0.025 & mlcopies $^{-1} d^{-1}$ \\
\cline { 3 - 3 }$U_{\max }$ & Maximum saturation of CD4 & & \\
\cline { 2 - 4 } & & $\in[0,1]$ & \\
\hline
\end{tabular}

Note: Table lis a modification of validated models of [18, 20, 21]

Table 2. Summary of constants and parameter values of model (3)

\begin{tabular}{|c|c|c|c|}
\hline \multirow{2}{*}{$\begin{array}{l}\text { Parameter } \\
\text { symbols }\end{array}$} & Parameters and constants & \multirow{2}{*}{$\begin{array}{l}\text { Initial } \\
\text { values }\end{array}$} & \multirow[t]{2}{*}{ Units } \\
\hline & Description & & \\
\hline$b$ & Natural source of uninfected CD $4^{+} \mathrm{T}$ cells & 0.6 & cells. $\mu l^{-1} d^{-1}$ \\
\hline$g$ & Growth rate of uninfected $\mathrm{CD}^{+} \mathrm{T}$ cells & 0.04 & cells. $\mu l^{-1} d^{-1}$ \\
\hline$\alpha_{1}$ & Natural death rate of uninfected $\mathrm{CD} 4^{+} \mathrm{T}$ cells & 0.02 & $d^{-1}$ \\
\hline$q_{i=1,2}$ & Treatment control functions for $U_{T}, I_{T}, V, P$ & $q_{i} \in[0,1]$ & \\
\hline$h_{1}$ & Rate of viral load infection on uninfected $\mathrm{CD} 4^{+} \mathrm{T}$ cells & 0.0044 & cells. $\mu l^{-1} d^{-1}$ \\
\hline$h_{2}$ & Rate of pathogen infection on uninfected $\mathrm{CD}^{+} \mathrm{T}$ cells & 0.0016 & cells. $\mu l^{-1} d^{-1}$ \\
\hline$z_{v}$ & Replication rate of viral load by $I_{T}$ cells & 0.5 & $\begin{array}{l}\text { copiesml }^{-1} \text { cells }^{1} \\
\text { uld }^{-1}\end{array}$ \\
\hline$z_{p}$ & Replication rate of pathogen by $I_{T}$ cells & 0.3 & $\begin{array}{l}\text { copiesml }^{-1} \text { cells } \\
\mu^{1} d^{-1}\end{array}$ \\
\hline$\alpha_{2}$ & Death rate of infected $\mathrm{CD} 4^{+} \mathrm{T}$ cells & 0.09 & $d^{-1}$ \\
\hline$\rho$ & $\begin{array}{l}\text { Clearance rate of infected cells by immune effectors } \\
\text { response }\end{array}$ & 2.5 & ulcells ${ }^{-1} d^{-1}$ \\
\hline$\alpha_{3}$ & Natural death rate of viral load & 0.04 & mlcopies $^{-1} d^{-1}$ \\
\hline$\alpha_{4}$ & Natural death rate of pathogen & 0.05 & mlcopies $^{-1} d^{-1}$ \\
\hline$c$ & CTLp proliferation & 0.005 & ulcells ${ }^{-2} d^{-1}$ \\
\hline$\lambda$ & CTLp differentiation & 0.006 & ulcells ${ }^{-1} d^{-1}$ \\
\hline$\alpha_{5}$ & Natural death rate of CTLp & 0.017 & $d^{-1}$ \\
\hline$\alpha_{6}$ & Natural death rate of CTLe & 0.006 & $d^{-1}$ \\
\hline$R_{0}$ & Growth rate of virulence & $10^{-7}$ & copies $^{-1}$ mld $^{-2}$ \\
\hline$\sigma_{1}$ & Optimal weight ratio $q_{1}$ & 10 & \\
\hline$\sigma_{2}$ & Optimal weight ratio $q_{2}$ & 100 & \\
\hline
\end{tabular}

Note: Table 2 is a clinical modification of [18, 20, 21] to accommodate the present novel dual HIV-pathogen model solvable using RK4 software

Epidemiological analysis of tables $(1 \& 2)$ in comparison to model $[18,20]$, reveal the critical role of susceptible growth rate $g$; the effect of treatment function $\left(1-q_{1}(t)\right)$, which inhibits further invasion by virions on healthy $\mathrm{CD} 4^{+} \mathrm{T}$ cells. Remarkably, the presence of logistic term in the first equation clearly defines the limit of $\mathrm{CD}^{+} \mathrm{T}$ cells under investigation. Furthermore, natural source of uninfected cells is seen been differentiated with respect to viral load and pathogen following the fact that infected T-cells once infected, is continuously infiltrated by virions. Next, been spur by the above analysis, we are bound to show that our state variables constitute set of living organisms and are therefore positive with bounded characterized solutions.

\subsection{Positivity of State Variables and Boundedness of Solution}

Suppose $S=D\left([-\tau, 0], \Re^{7}\right)$ be the Banach space of continuous mapping in the interval $[-\tau, 0]$ into 
$\mathfrak{R}^{7}$ equipped with the sup-norm (topology of uniform convergence). Then, from [22, 23], applying the fundamental theory of functional differential equations (FDEs), there exist unique solutions: $\left(u_{T}(t), i_{T}(t), v(\mathrm{t}), p(t), w(t), z(t), r(t)\right)$ to model (3) and having initial conditions

$$
\left(u_{T}(t), i_{T}(t), v(\mathrm{t}), p(t), w(t), z(t), r(t)\right) \in S .
$$

Biologically, these initial functions $u_{T}(\theta), i_{T}(\theta), v(\theta), p(\theta), w(\theta), z(\theta), r(\theta)$ are assumed to be nonnegative, i.e.

$$
\begin{aligned}
& u_{T}(\theta) \geq 0, i_{T}(\theta) \geq 0, v(\theta) \geq 0, p(\theta) \geq 0, \\
& w(\theta) \geq 0, z(\theta) \geq 0, r(\theta) \geq 0 \text { for all } \theta \in[-\tau, \theta]
\end{aligned} .
$$

Then, the positivity of state variables and boundedness of solution for model (3) with initial functions satisfying condition (4) and (5) is presented in the theorem hereof.

Theorem 2.1 Let $\left(u_{T}(t), i_{T}(t), v(\mathrm{t}), p(t), w(t), z(t), r(t)\right)$ be the solutions of model (3) satisfying conditions (4) and (5). Then $u_{T}(\theta), i_{T}(\theta), v(\theta), p(\theta), w(\theta), z(\theta), r(\theta)$ are all non-negative and bounded for all $t \geq 0$ of which solution exists.

Proof Invoking the result of (Thm. 2.1, p514-515, [23]), it is obvious to see from model (3) that

$$
\begin{aligned}
& u_{T}(t)=u_{(T)}(0) e^{-\int_{t_{0}}^{t_{f}}\left\{\alpha_{1}+g\left(1-\frac{u_{T}(\xi)+i_{T}(\xi)}{u_{\max }}\right)+\left(1-q_{1}(t)\right)\left[h_{1} v(\xi)-h_{2} p(\xi)\right] r(\xi)\right\} d \xi}
\end{aligned}
$$

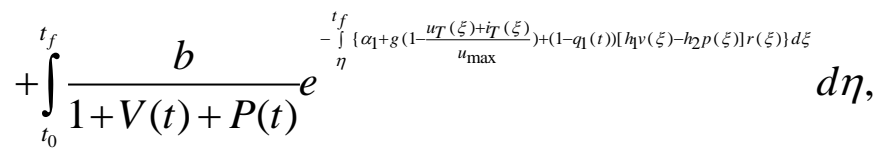

$$
\begin{aligned}
& i_{T}(t)=i_{t}(0) e^{-\int_{t_{0}}^{t_{f}}\left(\left(z_{v}+z_{p}\right) \alpha_{2}+\rho z(\xi)\right) d \xi}+\int_{t_{0}}^{t_{f}}\left(1-q_{1}(\mathrm{t})\right) r(\eta) u_{T}(\eta)\left[h_{1} v(\eta)-h_{2} p(\eta)\right] \\
& \cdot e^{-\int_{\eta}^{t_{f}}\left(\left(z_{v}+z_{p}\right) \alpha_{2}+\rho z(\xi)\right) d \xi} d \eta \\
& v(t)=v(0) e^{-\alpha_{3}(t)}+\int_{t_{0}}^{t_{f}}\left(1-q_{2}(t)\right) z_{v} \alpha_{2} i_{T}(\eta) e^{-\alpha_{3}(t)} d \eta, \\
& p(t)=p(0) e^{-\alpha_{4}(t)}+\int_{t_{0}}^{t_{f}}\left(1-q_{2}(t)\right) z_{p} \alpha_{2} i_{T}(\eta) e^{-\alpha_{4}(t)} d \eta, \\
& w(t)=w(0) e^{-\alpha_{5}(t)}+\int_{t_{0}}^{t_{f}}\left(c u_{T}(\xi) i_{T}(\xi)-\lambda i_{T}(\xi)-\alpha_{5}\right) d \xi, \\
& z(t)=z(0) e^{-\alpha_{6}(t)}+\int_{t_{0}}^{t_{f}}\left(\lambda i_{T}(\xi) w(\xi)-\alpha_{6}\right) d \xi
\end{aligned}
$$

and

$$
r(t)=r(0) e^{-q_{1}(t)}+\int_{t_{0}}^{t_{f}}\left(r_{0}(\xi)-q_{1}\right) d \xi .
$$

Positivity immediately follows from the above integral forms and conditions (4) and (5) satisfied.

For boundedness of the solution, we define 
Quantitative Approximability of Optimal Control by Linear Programing Model for Asymptomatic Dual HIV - Pathogen Infections

$$
U(t)=c\left(z_{v}+z_{p}\right) u_{T}(t) r(t)+c\left(z_{v}+z_{p}\right) i_{T}(t)+\frac{c}{2}(v(t) p(t))+\left(z_{v}+z_{p}\right) \rho z(t)
$$

and $b=\min \left\{\alpha_{1}, \alpha / 2, \alpha_{3}, \alpha_{4}, \alpha_{5}, \alpha_{6}\right\}$. By non-negativity of the solution, it follows that

$$
\begin{aligned}
& \frac{d}{d t}[U(t)]=c\left(z_{v}+z_{p}\right)\left[\frac{b}{1+v(t)+p(t)}+g u_{T}(\mathrm{t})\left(1-\frac{u_{T}(t)+i_{T}(t)}{u_{\max }(t)}\right)-\alpha_{1} u_{T}(t)-\left(h_{1} v(t) \cdot h_{2} p(t)\right) u_{T}(t) r(t)\right] \\
& +c\left(z_{v}+z_{p}\right)\left(h_{1} \cdot h_{2}\right) v(t) p(t) u_{T}(t) r(t)-\alpha_{2} c\left(z_{v}+z_{p}\right) i_{T}(t) \\
& -c\left(z_{v}+z_{p}\right) \rho \lambda i_{T}(t) w(t) z(t)+\frac{\alpha_{2} c\left(z_{v}+z_{p}\right)}{2} i_{T}(t) \\
& -\frac{c\left(\alpha_{3}+\alpha_{4}\right)}{2} v(t) p(t)+c\left(z_{v}+z_{p}\right) \rho \lambda i_{T}(t) w(t) z(t) \\
& -\left(z_{v}+z_{p}\right) \rho \lambda\left(\alpha_{5}+\alpha_{6}\right) w(t) z(t)-\left(z_{v}+z_{p}\right) r_{o}(t) r(t) \\
& =c\left(z_{v}+z_{p}\right) \frac{b}{1+v(t)+p(t)}-c \alpha_{1}\left(z_{v}+z_{p}\right) u_{T}(t)-\frac{\alpha_{2}}{2} c\left(z_{v}+z_{p}\right) i_{T}(t) \\
& -\frac{c\left(\alpha_{3}+\alpha_{4}\right)}{2} v(t) p(t)-\left(z_{v}+z_{p}\right) \rho \lambda\left(\alpha_{5}+\alpha_{6}\right) w(t) z(t) \\
& <c\left(z_{v}+z_{p}\right) \frac{b}{1+v(t)+p(t)}-b U(t) .
\end{aligned}
$$

This implies that $U(t)$ is bounded and so are $u_{T}(t), i_{T}(t), v(t), p(t), w(t), z(t)$ and $r(t)$. Hence, this completes the proof.

Remark 5 The consequences of Thm. 2.1 in conjunction to conditions (4) and (5) is that if $i_{T}(0)>0$ or $\{v(0), p(0)\}>0$, then $u_{T}(t), i_{T}(t), v(t), p(t), w(t), z(t)$ and $r(t)$ are actually positive. Furthermore, the boundedness as validated in Thm. 2.1 ensures the existence of solution for all $t \geq 0$.

At this point, we next validate the inclusion of two chemotherapy control measures and the crucial role of dual CTLs as immune system enhancement agent. The optimality control immediately comes to bear.

\section{OPTimal Control Problem for QCT}

For a quadrupled chemotherapy treatment (QCT), we invoke the epidemiological investigation of model [9], where minimum count of $\mathrm{CD}^{+} \mathrm{T}$ cells for an infected patient with which treatment is bound to commence is at $t_{0}(3)=0.25 \mathrm{~mm}^{3}$ for $t \in[3,30]$ months. Moreso, in the absence of medication transmutation of HIV to full-blown AIDS for infected patient is bound to occur if CD4 $4^{+} \mathrm{T}$ cells count fall below $\mathrm{CD}^{+}{ }_{\text {AIDS }}$ count of $<200 \mathrm{cell} / \mu \mathrm{l}$.

Accounting for this sort of precarious situation, the present study is therefore prime with the proposition of treatment regimen that aim at maximizing asymptomatic stage of dual HIV-pathogen infections. This is to say that we seek to maximize the performance index, which is the benefits base on $\mathrm{CD}^{+} \mathrm{T}$ cells and CTLs (= CTLp + CTLe) levels with ascertained minimization of systemic cost. In a balance situation, suppose HIV-pathogen infection transmute to AIDS after $t_{f}$, then we are confronted with the equation

$$
U_{T}(t)=C D 4^{+}{ }_{A I D S}, U_{T}(t) \geq C D 4^{+}{ }_{A I D S}, \forall t \in\left[t_{0}, t_{f}\right] .
$$


Quantitative Approximability of Optimal Control by Linear Programing Model for Asymptomatic Dual HIV - Pathogen Infections

Now, suppose systemic cost of chemotherapy is proportional to $q_{1}^{2}(t)$ and $q_{2}^{2}(t)$ for all $t \in\left[t_{0}, t_{f}\right]$ then the overall cost of treatment, which defines the objective functional, is given by

$$
J\left(q_{1}, q_{2}\right)=\int_{t_{0}}^{t_{f}}\left\{U_{T}(t)+Z(t)+W(t)-\left[\sigma_{1}\left(q_{1}(t)\right)^{2}+\sigma_{2}\left(q_{2}(t)\right)^{2}\right]\right\} d t
$$

where $\sigma_{i=1,2} \leq 1$ are the optimal weight factors necessary to balance the variation of drug toxicity. Furthermore, accounting for emergence of drug resistance, we observe that fixing a maximum cost of chemotherapy regimen amount to restricting the quantity of chemotherapeutic application. This is to say that the limit of chemotherapy should be sufficiently small positive integer. If we let this integer be $\tau$, such that risk of drug resistivity is sufficiently ignored, then equation (7) can be rewritten as:

$$
J\left(q_{1}, q_{2}\right)=\int_{t_{0}}^{t_{f}}\left\{U_{T}(t)+Z(t)+W(t)-\left[\sigma_{1}\left(q_{1}(t)\right)^{2}+\sigma_{2}\left(q_{2}(t)\right)^{2}\right]\right\} d t \leq \tau
$$

Setting $y_{i}=\left(u_{T}, i_{T}, v, p, w, z, r\right), i=1, \ldots . ., 7$ and $q(t)=\left(q_{1}(t), q_{2}(t)\right)$ the differential equations of model (3) can be represented as:

$$
\dot{y}(t)=j\left(t, y_{i}(t), q(t)\right)=\left(\begin{array}{c}
\frac{b}{1+y_{3}+y_{4}}+g\left(1-\frac{y_{1}+y_{2}}{y_{\max }}\right) y_{1}-\alpha_{1} y_{1}\left(1-q_{1}\right)\left[h_{1} y_{4}+h_{2} y_{5}\right] y_{1} y_{7} \\
\left(1-q_{1}\right)\left[h_{1} y_{4}+h_{2} y_{5}\right] y_{1} y_{7}-\left(z_{v}+z_{p}\right) \alpha_{2} y_{2}-\rho y_{2} y_{6} \\
\left(1-q_{2}\right) z_{v} \alpha_{2} y_{2}-\alpha_{3} y_{3} \\
\left(1-q_{2}\right) z_{p} \alpha_{2} y_{2}-\alpha_{4} y_{4} \\
c y_{1} y_{2} y_{5}-\lambda y_{2} y_{5}-\alpha_{5} y_{5} \\
\lambda y_{2} y_{5}-\alpha_{6} y_{6} \\
R_{0}-q_{1} R
\end{array}\right)
$$

Therefore, from equations (7), (8) and (9), the optimal chemotherapy control (regimen) problem can be derive as:

$$
\max _{q, t_{f}} \int_{t_{0}}^{t_{f}} d t
$$

subject to

$$
\left.\begin{array}{c}
\dot{y}=j(t, y, q) \\
\int_{t_{0}}^{t_{f}}\left\{u_{T}(t)+z(t)+w(t)-\left[\sigma_{1}\left(q_{1}\right)^{2}+\sigma_{2}\left(q_{2}\right)^{2}\right]\right\} d t \leq \tau \\
y_{1}\left(t_{0}\right)=y_{0}, y_{1}\left(t_{f}\right)=C D 4^{+}{ }_{A I D S} \\
y_{1}(t) \geq C D 4^{+}{ }_{A I D S}, t \in\left[t_{0}, t_{f}\right]
\end{array}\right\}
$$

Equations (10) and (11) represent a linear optimal control time problem (LOCTP)*.

For most cases, control function $q(\cdot)$ and corresponding state $y(\cdot)$ for final time $t_{f}$ that satisfy equation (11) may not exist. Then, overcoming such constraints, the problem of an LOCTP is conveniently solve using linear programing approach known as measure space. 


\section{Linear Programming Model For LOCTP}

The appreciation of a linear programing approach for an LOCTP requires the transformation of the LOCTP to a functional space, which is further transform to a measure space of infinite dimensional linear programing problem. Finally, the solution of this problem is then approximated by the solution of a finite dimensional LP of sufficiently large dimension. This classical measure theory for the computation of optimal control problem was first adopted by [24], applied by [25, 26] and extensively improved by [27]. Of note, model [18] recently applied the method in the calculation of time optimal control problem (TOCP), which led to the approximation of linear programing model.

\subsection{Transformation of LOCTP to Functional Space}

Let the compact set $X=X_{1} \times \ldots \ldots \times X_{7} \subset \mathfrak{R}^{7}$ and $Q=Q_{1} \times Q_{2} \subset \mathfrak{R}^{2}$ be the respective values of the model state variables $y(\cdot)$ and control input $q(\cdot)$, such that we set $N=\left[t_{0}, t_{f}\right]$.

Definition 4.1 We define a triple $k=\left[t_{f}, y, q\right]$ to be admissible if the following conditions hold:

i. The vector function $y(\cdot)$ be absolutely continuous and contain in $X$ for all $t \in N$

ii. The function $q(\cdot)$ takes its values in the set $Q$ and is Lebesgue measurable on $N$

iii. The function $q$ satisfies in system (11), i.e. on $T^{0}$, the interior of $N$.

Then, we assume that the set of all admissible triple is non-empty and is denoted by $m$. Let $k$ be an admissible triple and $B$ be an open ball in $\Re^{7}$ containing $N \times X$ and $D^{\prime}(B)$ be the space of all realvalued continuous differential equation on it. Let $\delta \in D^{\prime}(B)$ and define $\delta^{g}$ as follows:

$$
\begin{aligned}
\delta^{g}(t, y(t), q(t)) & =\frac{d \delta(t, y(t))}{d t} \\
& =\sum_{n=1}^{7} \frac{\partial \delta(t, y(t))}{\partial y_{n}} g_{n}(t, y(t), q(t))+\frac{\partial \delta(t, y(t))}{\partial t}
\end{aligned}
$$

for each $[t, y(t), q(t)] \in \Omega$, where $\Omega=N \times X \times Q$. The function $\delta^{g}$ is in the space $D(\Omega)$, the set of all continuous functions on the compact $\operatorname{set} \Omega$. Since $k=\left[t_{f}, y, q\right]$ is an admissible triple, we obtain

$$
\int_{t_{0}}^{t_{f}} \delta^{g}(t, y(t), q(t)) d t=\delta\left(t, y\left(t_{f}\right)\right)-\delta\left(t_{0}, y\left(t_{0}\right)\right)=\Delta \delta
$$

for all $\delta \in D^{\prime}(B)$.

Let $H\left(N^{0}\right)$ be the space of infinitely differentiable all real-valued function with compact support in $N^{0}$. Define:

$$
B^{n}(t, y(t), q(t))=y_{n}(t) \delta^{\prime}(t)+g_{n}\left(t, y(t), q(t) \beta(t), n=1, \ldots, 7 \forall \beta \in H\left(N^{0}\right)\right.
$$

Then, if $k=\left[t_{f}, y, q\right]$ be admissible triple for $n=1, \ldots, 7$ and $\forall \beta \in H\left(N^{0}\right)$, by equation (14), we obtain

$$
\begin{array}{r}
\int_{t_{0}}^{t_{f}} \beta^{n}(t, y(t), q(t)) d t=\int_{t_{0}}^{t_{f}} y_{n}(t), \beta^{\prime}(t) d t+\int_{t_{0}}^{t_{f}} g_{n}(t, y(t), q(t)) \beta(t) d t \\
=\left.y_{n}(t) \beta(t)\right|_{t_{0}} ^{t_{f}}+\int_{t_{0}}^{t_{f}} y_{n}(t), \beta^{\prime}(t) d t+\int_{t_{0}}^{t_{f}} g_{n}(t, y(t), q(t)) \beta(t) d t
\end{array}
$$


Quantitative Approximability of Optimal Control by Linear Programing Model for Asymptomatic Dual HIV - Pathogen Infections

since the function $\beta(\cdot)$ has compact support in $N^{0}$, so $\beta\left(t_{0}\right)=\beta\left(t_{f}\right)=0$ and $\dot{y}_{n}=g_{n}$. So,

$\int_{t_{0}}^{t_{f}} \beta^{n}(t, y(t), q(t)) d t=0$.

Furthermore, by adopting the functions of time dependent, we have:

$\int^{t_{f}} \eta(t, y(t), q(t)) d t=a_{\eta}, \forall \eta \in D^{\prime}(\Omega)$

where $D^{1}(\Omega)$ is the space of all functions in $D(\Omega)$ that depend only on time linearly and $a_{\eta}$ is the integral of $\eta$ on $N$. Equations (13), (15) and (16) are really weak form of the first, third and fourth equations of equation (11). It's obvious that third constraint of equation (11) is considered on the right side of equation (13) with functions $\delta \in D^{\prime}(B)$, which are monomials of $y_{1}$. Similarly, the fourth constraint is considered by appropriate definition of set $X$. Then, we can now consider the following linear functional on $D(\Omega)$. Define:

$$
\Gamma_{k}: G \rightarrow \int_{\eta} G(t, y(t), q(t)) d t, \forall G \in D(\Omega)
$$

Proposition 4.1 The transformation $k \rightarrow \Gamma_{k}$ of admissible triple in $M$ into the linear mappings $\Gamma_{k}$ defined in (17) is an injection.

Proof Here, we start by showing that if $k_{1} \neq k_{2}$, then $\Gamma_{k_{1}} \neq \Gamma_{k_{2}}$. Let $k_{n}=\left[t_{f}, y_{n}, q_{n}\right], n=1,2$ be different admissible triples. If $t_{f_{1}}=t_{f_{2}}$ then there is a subinterval of $\left[t_{0}, t_{f}\right]$, say $n_{1}$, where $y_{1}(t) \neq y_{2}(t)$ for each $t \in n_{1}$. A continuous function $G$ can be constructed on $\Omega$ so that the right-hand side of (17) corresponding to $k_{1}$ and $k_{2}$ are not equal. In the same requirement, assume $G$ b is independent of $q$ such that foe all $t \in N_{1}$, the function is non-negative for the graph of $y_{1}(t)$ but zero on $y_{2}(t)$, then the linear functional are not equal. In other words, if $t_{1} \neq t_{2}$, then $\Gamma_{k_{1}}$ and $\Gamma_{k_{2}}$ have different domains and are not equal.

Thus, the LOCTP of (10)-(11) is converted to the following optimization problem in functional space:

$$
\text { Maximize } \Gamma_{k}(1) \quad(\text { from (10)) }
$$

Subject to

$$
\begin{aligned}
& \Gamma_{k}\left(\psi^{g}\right)=\Delta \psi, \psi \in D^{\prime}(B) \\
& \Gamma_{k}\left(\beta^{n}\right)=0, n=1, \ldots 7, \beta \in H\left(N^{0}\right) \\
& \Gamma_{k}(\eta)=a_{\eta}, \eta \in D^{\prime}(\Omega) \\
& \Gamma_{k}(E) \leq \tau
\end{aligned}
$$

where $E(t, y(t), q(t))=u_{T}(t)+z(t)+w(t)-\left[\sigma_{1}\left(q_{1}(t)\right)^{2}+\sigma_{2}\left(q_{2}(t)\right)^{2}\right]$ and equation (19) generated from equations $(13,15,16$ and 10$)$ respectively.

\subsection{Transformation to Measure Space}

Let $L^{+}(\Omega)$ denotes the space of all non-negative Radon measures on $\Omega$. By the Riez representation theorem, there exists a unique positive Radon measure $\mu$ on $\Omega$ such that:

$$
\Gamma_{k}(G)=\int_{N} G(t, y(t), q(t)) d t=\int_{\Omega} G(t, y, q) d \mu \equiv \mu(G), G \in(\Omega) .
$$

Then, we can transform the space of optimization problem to measure space. Conveniently, this implies that optimization problem in functional space (18)-(19) can be transform to the following new problem in measure space: 


$$
\underset{\mu \in L^{+}(\Omega)}{\operatorname{Maximize}} \mu(1)
$$

Subject to

$$
\begin{aligned}
& \mu\left(\delta^{g}\right)=\Delta \delta, \delta \in D^{\prime}(B) \\
& \mu\left(\beta^{n}\right)=0, n=1, \ldots 7, \beta \in H\left(N^{0}\right) \\
& \mu(\eta)=a_{\eta}, \eta \in D^{1}(\Omega) \\
& \mu(E) \leq \tau
\end{aligned}
$$

Thus, we see at once that we're now considering the maximization of (21) over a set of all positive Radon measure on $\Omega$ denoted by $A$ and which satisfies equation (22).

Remark $6 \mathrm{We}$ opt for the exploration of this measure theoretical approach for the problem following the existence of an optimal measure in the set $A$, which can be solve in a straightforward manner without having imposition of conditions of artificial convexity. The following theorem further buttresses the above assertion.

Theorem 4.1 Revoking ([18], Thm. 4.2.1, p.52)

The measure theoretical problem of maximizing (21) with equality and inequality constraints (22) has an optimal solution $\mu^{*}$.

Proof Here, we show that second and third equations of (22) are special version of the first equation of (22). Therefore, the set $A$ can be written as $A=A_{1} \cap A_{2}$, where

$$
A_{1}=\bigcap_{\delta \in D^{\prime}(B)}\left\{\mu \in L^{+}(\Omega): \mu\left(\delta^{g}\right)=\Delta \psi\right\}
$$

and

$$
A_{2}=\left\{\mu \in L^{+}(\Omega): \mu(E) \leq \tau\right\}
$$

Assume that $k=\left[t_{f}, y, q\right]$ is an admissible triple. It is well-known that the set $\left\{\mu \in L^{+}(\Omega): \mu(1)=t_{f}-t_{0}\right\}$ is compact in weak* - topology. Furthermore, $A_{1}$ as intersection of inverse image of closed singleton set $\{\Delta \delta\}$ under continuous function $\mu \rightarrow \mu\left(\delta^{g}\right)$ is also closed. It can be shown in a similar way that $A_{2}$ is closed. Thus, $A$ is a close subset of a compact set. This proves the compactness of the set $A$. Since the functional as intersection $\mu \rightarrow \mu(1)$ mapping the compact set $A$ on the real line is continuous and so has a maximum on the compact set $A$.

Next, following the analysis in [26], the problem (21)-(22) is approximated by a LP problem and a triple $k^{*}$, which approximate the action of $\mu^{*} \in A$ is achieved.

\subsection{Approximation for Measure space Problem}

Of interest, the problem in measure space (21)-(22) is an infinite dimensional linear programing problem of which all the functions in (22) are linear with respect to measure $\mu$. This is obvious following the fact that $L^{+}(\Omega)$ is infinite dimensional space. Remarkably, approximation of solution of this problem is overcome by the solution of a finite dimensional LP of sufficiently large dimension. Then, from the solution of this new finite dimensional LP, we induce an approximated admissible triple in a suitable manner.

The simplification of these processes requires us to construct an intermediate problem still infinitedimensional for (21) but not over the set $A$. Rather, over a subset $L^{+}(\Omega)$ and having only a finite 
Quantitative Approximability of Optimal Control by Linear Programing Model for Asymptomatic Dual HIV - Pathogen Infections

numbers of constraints with (22) being satisfied. We achieve this by choosing countable sets of functions whose linear combinations are dense in the sets $D^{\prime}(B), D^{1}(\Omega)$ and $H\left(N^{0}\right)$ from which we then select a finite number of them.

Assume the set $\left\{\delta_{i}: i=1,2, \ldots ..\right\}$ be such that the linear combinations of the functions $\delta_{i} \in D^{\prime}(B)$ are uniformly dense in $D^{\prime}(B)$. For instance, these functions can be taken to be monomials in $t$ and the components of the vector $y$. Next, we show that these monomials are suitable for our problem i.e.

$$
t^{i} y_{1}^{n}=y_{1}^{n} y_{h}^{i}, i \in\{0,1\}, n \in\{1,2, \ldots . .\}, h \in\{2,3, . ., 7\} .
$$

We $\operatorname{set}\left\{\beta_{i}: i=1,2, \ldots \ldots\right\}$ such that the linear combinations of the functions $\beta_{i} \in H\left(N^{0}\right)$ are uniformly dense in $H\left(N^{0}\right)$. For $s=1,2, \ldots \ldots$ with some function designated as [27]:

$$
\beta_{2 s-1}(t)=\left\{\begin{array}{cc}
\sin \left(\frac{2 \pi s\left(t-t_{0}\right)}{\Delta T}\right) & t \leq t_{1} \\
0 & \text { otherwise }
\end{array}\right.
$$

and

$$
\beta_{2 s}(t)=\left\{\begin{array}{cc}
1-\cos \left(\frac{2 \pi s\left(t-t_{0}\right)}{\Delta T}\right) & t \leq t_{1} \\
0 & \text { otherwise }
\end{array}\right.
$$

where $\Delta T=t_{1}-t_{0}$ and $t_{1}$ is a lower bound for optimal time, which can be obtain via controllability.

Finally, let the $\operatorname{set}\left\{\eta_{i}: i=1,2, \ldots \ldots\right\}$ be such that the linear combinations of the functions $\eta_{i} \in D^{1}(\Omega)$ are uniformly dense in $D^{1}(\Omega)$. These functions are monomials in $t$ and are given as:

$\eta_{w}(t)=t^{w}, w=0,1,2 \ldots \ldots \ldots$

Remark 7 From the basis of (12) and (14), it is obvious that second and third equations of (11) are also derived from the first equation of (11) if we set $\delta(t, y(t))=y_{n}(t) \beta(t)$ and $\delta(t, y(t))=\int_{0}^{t} \eta(\xi) d \xi$ respectively.

Therefore, completion of approximation approach for the measure space is subject to the following 3 propositions:

Proposition 4.2 Assuming the linear program is consisting of maximizing function $\mu \rightarrow \mu(1)$ over the $\operatorname{set} U_{L}$ of measures in $L^{+}(\Omega)$ and satisfying: $\mu\left(\delta_{i}^{g}\right)=\Delta \delta_{i}, i=1, \ldots \ldots, L$ and $\mu(E) \leq \tau$. Then $\gamma_{L} \equiv \max _{U_{L}} \mu(1)$ tends to $\gamma \equiv \max _{U} \mu(1)$ as $L \rightarrow \infty$.

Proof Given that $U_{1} \supseteq U_{2} \supseteq \ldots . . U_{L} \supseteq \ldots \ldots . . U$, then $\gamma_{1} \geq \gamma_{2} \geq \ldots \ldots . \gamma_{L} \geq \ldots . . \geq \gamma$. Hence, $\left\{\gamma_{r}\right\}$ is non-increasing and bounded sequence, which converges to the number $\lambda$, such that $\lambda \geq \gamma$.

Set $R \equiv \bigcap_{L=1}^{\infty} U_{L}$, then $R \supseteq U$ and $\lambda \equiv \max _{R} \mu(1)$. It is sufficient to show $R \subseteq U$. Assume $\mu \in R$ and $\delta \in D^{\prime}(B)$. Since linear combinations of the functions $\left\{\delta_{n}, n=1,2, \ldots \ldots\right\}$ are uniformly dense in $D^{\prime}(B)$, there is the sequence $\left\{\tilde{\delta}_{p}\right\} \in \operatorname{span}\left\{\delta_{n}, n=1,2, \ldots \ldots\right\}$ such that $\tilde{\delta}_{p}$ tends to $\delta$ uniformly as $p \rightarrow \infty$. Hence, $W_{1}, W_{2}$ and $W_{3}$ tends to zero as $p \rightarrow \infty$ where $W_{1}=\sup \left|\delta_{y}-\tilde{\delta}_{p_{y}}\right|, W_{2}=\sup \left|\delta_{t}-\tilde{\delta}_{p_{t}}\right|$ 
Quantitative Approximability of Optimal Control by Linear Programing Model for Asymptomatic Dual HIV - Pathogen Infections

and $W_{3}=\sup \left|\delta-\tilde{\delta}_{p}\right|$. Then, we have $\mu \in R$ and functional $g \rightarrow \mu(g)$ is linear. Therefore, $\mu\left(\tilde{\delta}_{p}^{g}\right)=\Delta \tilde{\delta}_{p}$ and $\left|\mu\left(\delta^{g}\right)-\Delta \delta\right|=\left|\mu\left(\delta^{g}\right)-\Delta \delta-\mu\left(\tilde{\delta}_{p}\right)+\Delta \tilde{\delta}_{p}\right|$ $=\left|\int_{\Omega}\left\{\left[\delta_{y}(t, y)-\tilde{\delta}_{p_{y}}(t, y)\right] g(t, y, q)+\left[\delta_{t}(t, y)-\tilde{\delta}_{p_{t}}(t, y)\right]\right\} d \mu-\left(\Delta \delta-\Delta \tilde{\delta}_{p}\right)\right|$

$$
\leq W_{1} \int_{\Omega}|g(t, y, q)| d \mu+W_{2} \int_{\Omega} d \mu+2 W_{3} .
$$

Since the R.H.S. of the above inequality tends to zero as $p \rightarrow \infty$, while L.H.S. is independent of $p$, then $\mu\left(\delta^{g}\right)=\Delta \delta$. Thus, $R \subseteq U$ and $\lambda \geq \gamma$, which implies $\lambda=\gamma$.

Proposition 4.3 The measure optimal solution $\mu *$ in the $\operatorname{set} U_{L}$ at which the functional $\mu \rightarrow \mu(1)$ attains its maximum has the form

$$
\mu^{*}=\sum_{n=1}^{L+1} \alpha_{n}^{*}\left(z_{n}^{*}\right)
$$

where $\alpha_{n}^{*} \geq 0, z_{n}^{*} \geq 0$ and $\varphi(z)$ is unitary atomic measure with the support being the $\operatorname{singleton} \operatorname{set}\left\{z_{n}^{*}\right\}$, characterized by $\varphi(z)(G)=G(z), z \in \Omega$.

Proof In restricting our attention to finding measure in the form $\mu=\sum_{n=1}^{L+1} \alpha_{n} \varphi(z)$, which maximizes functional $\mu \rightarrow \mu(1)$ and satisfies last equation (22) and $L$ number of constraints in the form of firstthird of equation (22), we invoke the result of appendix of [25]. Clearly, $\mu(G)=\sum_{n=1}^{L+1} \alpha_{n} G\left(z_{n}\right), \forall G \in D(\Omega)$. Therefore, by choosing $L$ number of functions in the form of (23), $W$ number of functions in the form of (24), which leads to $L_{2}=7 L_{2}^{\prime}$ number of functions of the kind (16) for which we have numbers sequentially as $\wp_{h}, h=1, \ldots \ldots . ., L_{2}$. Then, the infinite dimensional problem (21)-(22) is approximated with the aid of finite dimensional non-linear programing (NLP) problem:

$$
\underset{\alpha_{n} \geq 0, z_{n} \in L^{+}(\Omega)}{\operatorname{aximize}} \sum_{n=1}^{L+1} \alpha_{n}
$$

Subject to

$$
\begin{gathered}
\sum_{n=1}^{L+1} \alpha_{n} \delta_{i}^{g}\left(\mathrm{z}_{n}\right)=\Delta \delta_{i}, i=1, \ldots, L_{1} \\
\sum_{n=1}^{L+1} \alpha_{n} \biguplus_{h}\left(\mathrm{z}_{n}\right)=0, h=1, \ldots, L_{2} \\
\sum_{n=1}^{L+1} \alpha_{n} \eta_{w}\left(\mathrm{z}_{n}\right)=a_{\eta}, w=1, \ldots, W \\
\sum_{n=1}^{L+1} \alpha_{n} E\left(\mathrm{z}_{n}\right) \leq \tau
\end{gathered}
$$

where $L=L_{1}+L_{2}+W$. We are then confronted with NLP with more than $2(L+1)$ unknown $\alpha_{n}, z_{n}, n=1, \ldots ., L+1$. 
Quantitative Approximability of Optimal Control by Linear Programing Model for Asymptomatic Dual HIV - Pathogen Infections

Finally, using the finite dimensional linear programing problem, the following last proposition guarantees the approximation of the problem.

Proposition 4.4 Let $\Omega_{j}=\left\{x_{1}, x_{2}, \ldots \ldots, x_{j}\right\}$ be a countable dense subset of $\Omega$, for any $J$ sufficiently large number. Given $\varepsilon \geq 0$, a measure $\omega \in L^{+}(\Omega)$ can be found such that

$$
\begin{aligned}
& \left|\omega\left(\delta_{i}^{g}\right)-\mu^{*}\left(\delta_{i}^{g}\right)\right| \leq \varepsilon, i=1, \ldots . L_{1} \\
& \left|\omega\left(\mathrm{ю}_{h}\right)-\mu^{*}\left(\delta_{h}\right)\right| \leq \varepsilon, h=1, \ldots . L_{2} \\
& \left|\omega\left(\eta_{w}\right)-\mu^{*}\left(\eta_{w}\right)\right| \leq \varepsilon, w=1, \ldots . W \\
& \left|\omega(E)-\mu^{*}(E)\right| \leq \varepsilon
\end{aligned}
$$

where $\omega$ is a measure with the form:

$$
\omega=\sum_{n=1}^{L+1} \alpha_{n}^{*} \varphi\left(z_{n}\right)
$$

and having the coefficient $\alpha_{n}^{*}, n=1, \ldots ., \mathrm{L}+1$ been the same as optimal measure (26) and $z_{n} \in U_{J}, n=1, \ldots ., L+1$.

Proof Suppose functions $\delta_{i}^{g}{ }^{\prime} s, \wp_{h}{ }^{\prime} s, \omega_{s}{ }^{\prime} s$ and $E$ are sequentially rename as $G_{n}, n=1, \ldots \ldots, L+1$. Then for $n=1, \ldots \ldots, L+1$,

$$
\begin{gathered}
\left|\left(\mu^{*}-\omega\right) G_{n}\right|=\left|\sum_{n=1}^{L+1} \alpha_{n}^{*}\left[G_{n}\left(z_{n}^{*}\right)-G_{n}\left(z_{n}\right)\right]\right| \\
\leq\left(\sum_{n=1}^{L+1} \alpha_{n}^{*}\right) \max _{i, n}\left|G_{n}\left(z_{n}^{*}\right)-G_{n}\left(z_{n}\right)\right|
\end{gathered}
$$

with $G_{n}{ }^{\prime} s$ continuous. Therefore, $\max _{i, n}$ can be made less than $\frac{\varepsilon}{\sum_{n=1}^{L+1} \alpha_{n}^{*}}$ by choosing $z_{i}, i=1, \ldots ., L+1$, sufficiently near $z_{i}^{*}$. We then construct the dense subset $\Omega_{J}$, such that $N$ is divided into $W$ subintervals as follows:

$$
N_{w}=\left[t_{0}+\frac{(w-1) \Delta T}{W-1}, t_{0}+\frac{w \Delta T}{W-1}\right), w=1,2, \ldots \ldots, W-1
$$

and

$$
N_{w}=\left[t, t_{f}\right)
$$

Furthermore, the intervals $P_{i}{ }^{\prime} s$ and $Q_{n}{ }^{\prime} s$ are divided into $u_{i}$ and $v_{j}$ subintervals respectively. Then, the set $\Omega$ is divided into $J=W u_{1} u_{2} u_{3} u_{4} u_{5} u_{6} v_{1} v_{2}$ cells. One point is chosen from each cell, yielding a grid of points, which are numbered sequentially as $x_{n}=\left(t_{n}, y_{1_{n}}, \ldots . ., y_{7_{n}}, q_{1_{n}}, q_{2_{n}}\right), n=1, \ldots ., J$.

Remark 8 The function of the kind (25) can be conveniently approximated by a linear combination of characteristic function of subintervals of $N$. Therefore, in reality, we observe the function $\eta_{w}(t) \equiv \chi_{N_{w}}(t), w=1, \ldots ., W$ instead of the function (25), where $N_{w}{ }^{\prime} s$ are represented by (31) and $\chi_{N_{w}}$ denotes the characteristic function of $N_{w}$. This is obvious for the choice of $\eta_{w}{ }^{\prime} s$ because of it crucial role in construction of control functions [24, 25]. 
Quantitative Approximability of Optimal Control by Linear Programing Model for Asymptomatic Dual HIV - Pathogen Infections

Therefore, considering the equation (30), the NLP (27)-(28) is transformed to LP of the form:

$$
\underset{\alpha_{n} \geq 0}{\operatorname{Maximize}} \sum_{n=1}^{J} \alpha_{n}
$$

Subject to

$$
\begin{aligned}
& \sum_{n=1}^{J} \alpha_{n} \delta_{i}^{g}\left(\gamma_{n}\right)=\Delta \delta_{i}, i=1, \ldots ., L_{1}, \quad \sum_{n=1}^{u} \alpha_{n}=\frac{\Delta T}{W-1} \\
& \sum_{n=1}^{J} \alpha_{n} O_{h}\left(\gamma_{n}\right)=0, h=1, \ldots, L_{2}, \quad \sum_{n=(W-2) u+1}^{(W-1) u} \alpha_{n}=\frac{\Delta T}{W-1} \\
& \sum_{n=1}^{J} \alpha_{n} E\left(\gamma_{n}\right) \leq \tau, \quad \sum_{n=(W-1) u+1}^{W u} \alpha_{n}=t_{f}-t_{u}, \quad y_{i}\left(t_{f}\right) \in P_{i}, i=2,3,4,5,6,7
\end{aligned}
$$

where $u=\frac{J}{W}$. So, more importantly we need only to construct the function $q(\cdot)$, since $y(\cdot)$ is simply the corresponding solution of differential equations of the system (3), which can be numerically estimated.

Thus, by simplex method approach, nonzero optimal solution $\alpha_{i_{1}}^{*}, \alpha_{i_{2}}^{*}, \ldots . \alpha_{i_{k}}^{*}$ of LP (32)-(33) can be obtain with $k$ not exceeding the number of constraints i.e. $k \leq L_{1}+L_{2}+W+1$. By setting $\alpha_{i_{0}}^{*}=t_{0}$, piecewise control pair $r(t)=\left(r_{1}(t), r_{2}(t)\right)$, which thus approximate the action of the optimal control base on the nonzero coefficients i.e.

$$
q(t)=\left\{\begin{array}{cc}
\left(q_{i_{i_{n}}}, q_{2_{i_{n}}}\right) & t \in\left[\sum_{h=0}^{n-1} \alpha_{i_{h}}^{*}, \sum_{h=0}^{n} \alpha_{i_{h}}^{*}\right), n=1,2, \ldots, k \\
0 & \text { otherwise }
\end{array}\right.
$$

where $q_{1_{i_{n}}}$ and $q_{2_{i_{n}}}$ are respectively $8^{\text {th }}$ and $9^{\text {th }}$ components of $x_{i_{n}}$.

\section{NUMERICAL SimUlationS AND DisCUSSION}

In affirmation of our established model, we shall simulate a number of illustrative examples for a no treatment situation and then for the application of chemotherapy. This is followed by the analyses (discussion) of the achieved results.

\subsection{Numerical Simulations}

We set to perform a number of numerical simulations to account for our analysis in sections 2, 3 and 4 respectively. First, we illustrate the viability of the methodological application of treatment functions by considering the case when treatments were not initiated i.e. $q_{1}, q_{2}=0$. Then, the implication is that for a standard model of equation (3), we investigate the crucial role of natural adaptive immune effectors response and the intrinsic virulence of dual viruses.

For simplicity and compatibility with Runge-Kutter of order 4 in Mathcad surface, we convert the state variables to $\operatorname{read}\left\{U_{T}, I_{T}, V, P, W, Z, R\right\}=\sum_{i=1}^{7} H_{i}, i=1, . ., 7$, such that for $q_{1}, q_{2}=0$ and applying [9] with time interval of $\left[t_{0}, t_{f}\right]=(3,30]$ and model values as in tables $(1 \& 2)$, we investigate as depicted by fig. 2(a-d) an infected patient with only immune effectors response as the only anti-HIV antigens. 
Quantitative Approximability of Optimal Control by Linear Programing Model for Asymptomatic Dual HIV - Pathogen Infections

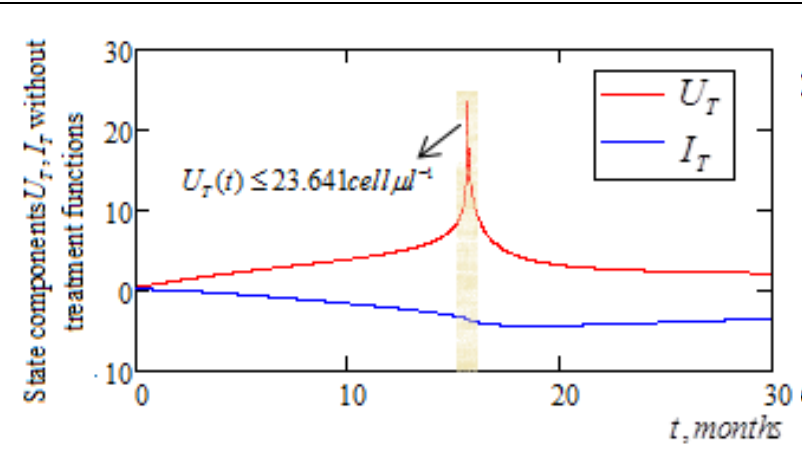

a) Simulation of uninfected and infected $\mathrm{CDA}^{+} \mathrm{T}$ cells with $q_{\mathrm{i}}=0, q_{2}=0$

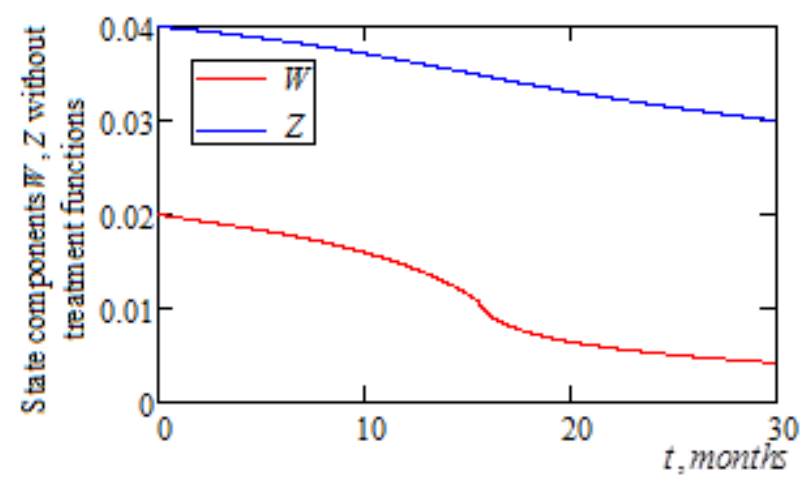

c) Simulation of immune effectors responses (CTLp and CTLe) with $q_{1}=0, q_{2}=0$

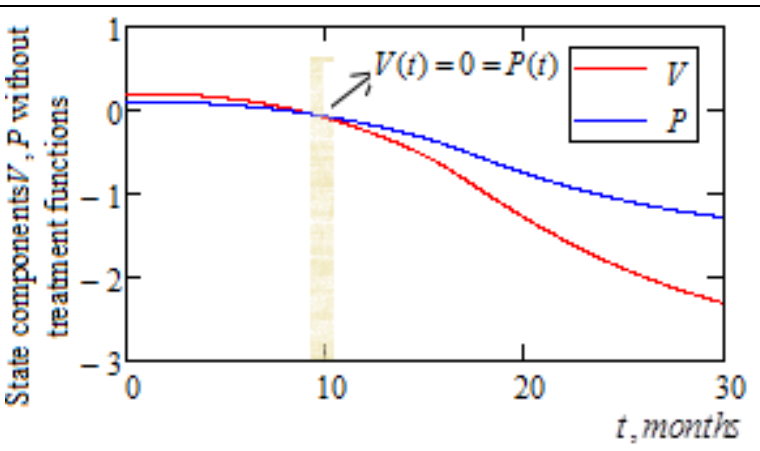

b) Simulation of infectious viral load and pathogen with $q_{1}=0, q_{2}=0$

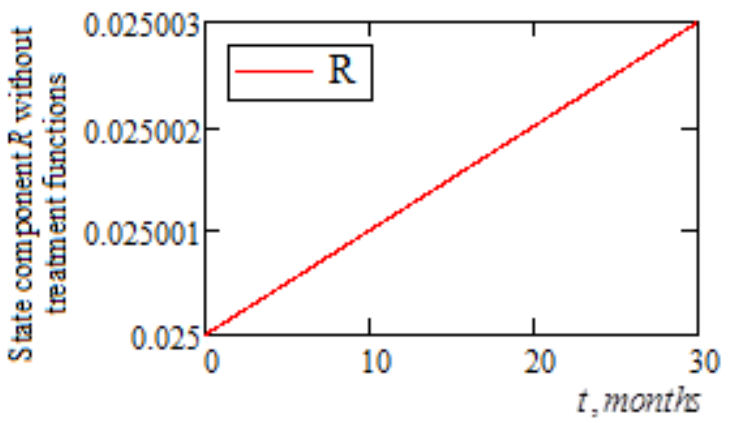

d) Simulation of intrinsic virulence with $r_{0}=10^{-7}$ and $q_{1}=0, q_{2}=0$

Fig. 2 (a-d) Simulation of untreated dual HIV-pathogen with CTLp $=0.2=C T L e$ and $q_{1}=0=q_{2}$

Specifically, fig. 2(a) depicts two key state components - the epidemiological behavior of healthy $\mathrm{CD} 4^{+} \mathrm{T}$ cells, $U_{T}(t)$ and the corresponding ravage infected $\mathrm{CD} 4^{+} \mathrm{T}$ cells $I_{T}(t)$. Here, we observe that the first function $U_{T}(t)$ exhibits gradual inclination with geometric peak value

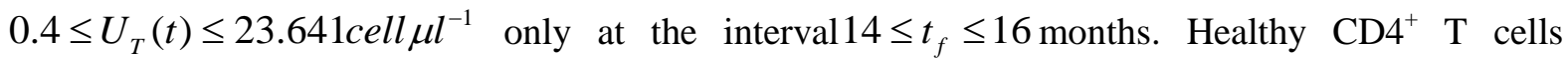
thereafter decline to minimal value of $U_{T}(t)=2.5 \mathrm{cell}_{\mu l^{-1}}$ for $16 \leq t_{f} \leq 30$ months. The second function denoting infected $\mathrm{T}$-cells are characterize by gradual decline due to active immune effectors response with minimal value $-4.364 \mathrm{cell}_{\mu} \mathrm{l}^{-1}$ at $18 \leq t_{f} \leq 21$ months and thereafter incline slightly to $I_{T}(t)=-3.72 \mathrm{cell} \mu l^{-1}$.

From fig. 2(b), we see infectious patient with high adaptive natural immune effectors response combating replication of virions at the early set-point of infection i.e. $t_{f} \leq 10$, following possible self-restriction and adherent to medicated conditions. Both virions (viral load and pathogen) achieve zero elimination at the time interval $t_{f} \geq 10$ months due to concentration of CTLp and CTLe. Fig. 2(c) reveals an undulating trajectory decline of precursors of CTLs with $0.01 \leq W(t) \leq 0.02 \mathrm{cell}_{\mu l^{-1}}$ at $t_{f} \leq 16$ months. The component further decline upon prolong observation with

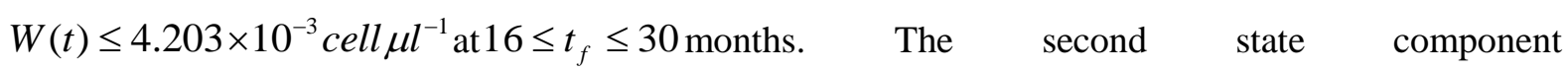
$Z(t) \leq 0.04 \mathrm{cell}_{\mu l^{-1}}$ representing active effectors of CTLs exhibits slight linear decline at initial period at $t_{f} \leq 16$ months with sharper decline to $Z(t) \leq 0.03$ cell $_{\mu l} l^{-1}$ at $16 \leq t_{f} \leq 30$ months. Furthermore, under the auspices of only adaptive immune effectors response, the aggressiveness of 
Quantitative Approximability of Optimal Control by Linear Programing Model for Asymptomatic Dual HIV - Pathogen Infections

virions (intrinsic virulence) remains slightly on the increase as depicted by fig. 2(d). Here, $R(t)$ increases from $R(t)=0.025 \rightarrow 0.025003$ mlcopies $^{-1} d^{-1}$ at $3 \leq t_{f} \leq 30$ months.

Further investigation is conducted following the introduction of treatment functions, which ensure that $q_{i=1,2} \geq 0$ and with parameter values as prescribed by tables $(1 \& 2)$. Clearly, we investigate the initiation of highly toxic chemotherapy at set-point, i.e. $q_{1}(t)=0.5, q_{2}(t)=0.3$, such that the optimal weight factors regulating these chemotherapies is given by $\sigma_{1}=10, \sigma_{2}=100$. Then, fig. 3(a-d) below represents the linear programing for treated asymptomatic dual HIV-pathogen infected patient under multiple chemotherapy and dual cytotoxic T-lymphocytes.

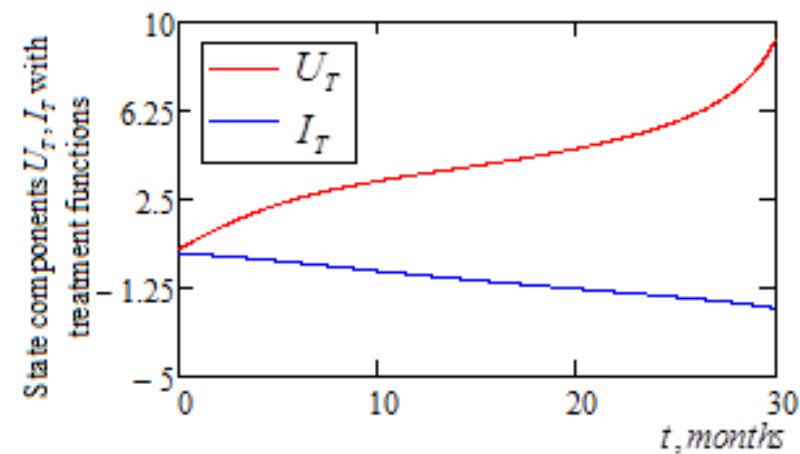

a) Simulation of uninfected and infected $\mathrm{CD4}^{+} \mathrm{T}$ cells with $q_{\mathrm{i}}=0.5, q_{2}=0.3$

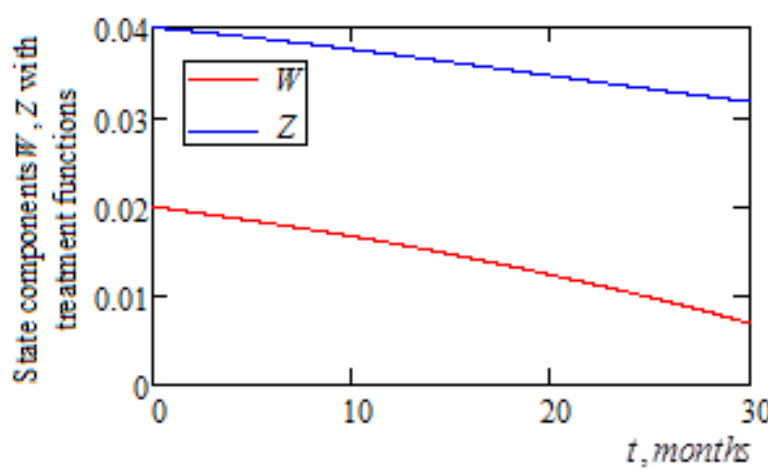

c) Simulation of immune effectors responses (CTL p and CTLe) with $q_{1}=0.5, q_{2}=0.3$

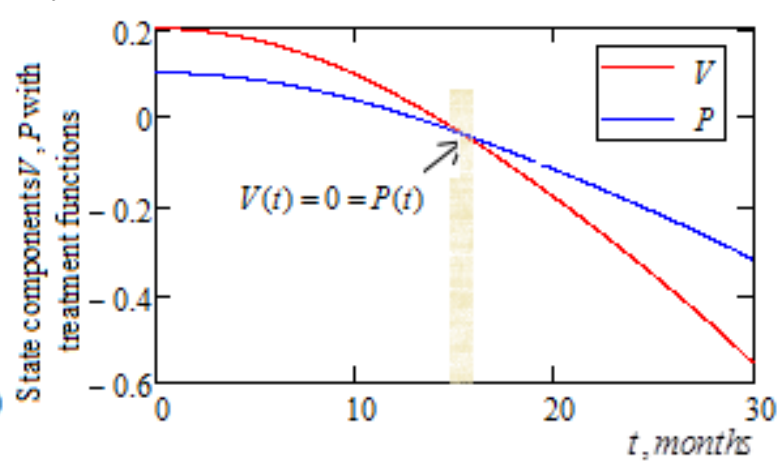

b) Simulation of infectious viral load and pathogen with $q_{\mathrm{i}}=0.5, q_{2}=0.3$

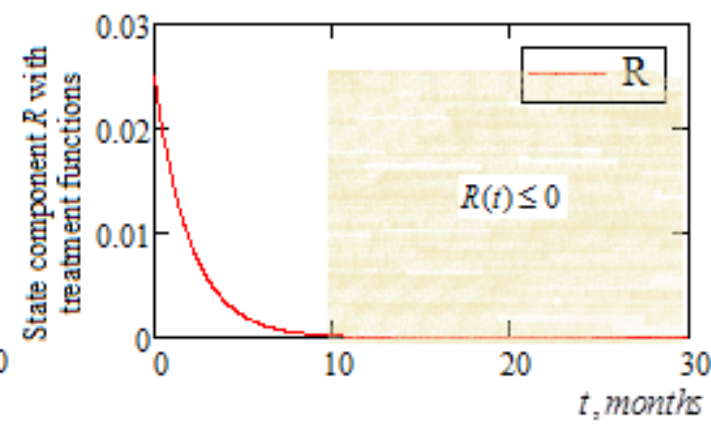

d) Simulation of intrinsic virulence with $r_{0}=10^{-7}$ and $q_{1}=0.5, q_{2}=0.3$

Fig.3(a-d) Behavioral dynamics of treated state components $U_{T}, I_{T}, V, P, W, Z, R$ with $q_{1}=0.5, q_{2}=0.3$

From fig. 3(a), we investigate the healthy and infected $\mathrm{CD}^{+} \mathrm{T}$ cells progression given the above medical conditions. In the first case for $U_{T}(t)$, we observe overall initial slight concave inclinatory trend in the interval $3 \leq t_{f} \leq 14$ months and then assume slant convex inclination achieving maximum value of $U_{T}(t) \leq 9.328 \mathrm{cell}_{\mu} \mathrm{l}^{-1}$ at $t_{f} \leq 30$ months. The second state function - the infected $\mathrm{CD} 4^{+} \mathrm{T}$ cells shows smooth linear decline of infectious particles to complete zero in the time interval $16 \leq t_{f} \leq 30$ months. In fig. 3(b), the first function representing viral $\operatorname{load} V(t)$ is seen to exhibit initial sharp concave declination with minimal zero value at $t_{f} \leq 16$ months. The second state function - parasitoid-pathogen $P(t)$, exhibits similar declining behavior with shallow initial decrease when compared to viral load. Of note, the elimination of both virions is achieved at $t_{f} \leq 16$ months (i.e. $t_{f} \equiv 480$ days $)$.

From fig. 3(c), we investigate the contributive roles of two sub-CTLs (CTLp and CTLe). The first state function $W(t)$ representing the passive immune precursor of CTLs exhibits slight concave

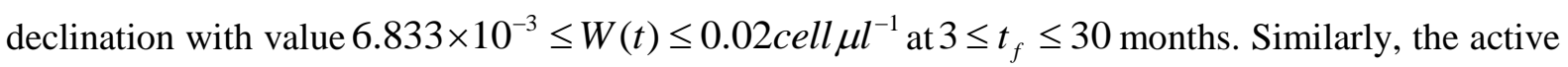


immune effectors of CTLs shows linear decline with value $0.032 \leq Z(t) \leq 0.04 \mathrm{cell}_{\mu l^{-1}}$ in the interval $3 \leq t_{f} \leq 30$ months of clinical investigation. Finally, from fig. 3(d), following the introduction of multiple chemotherapies functions and dual cytotoxic T-lymphocytes concentration, virions aggressiveness (intrinsic virulence) denoted by $R(t)$ is seen subdue to near zero value at $10 \leq t_{f} \leq 30$ months.

\subsection{Discussion}

The present study seek using ODEs the formulation of 7-Dimensional nonlinear mathematical dual HIV-pathogen dynamic model for the determination of time optimal control problem for a fast progressing asymptomatic HIV-pathogen infected patient. The treatment functions as applied in this study involve quadrupled treatment controls with model transformed to a time optimal control problem. The model analyses explored approximation approach of linear programing method followed by quantitative numerical simulations. The study is an explicit extension of related scientific investigations as was carefully highlighted in literature of this paper.

In the circumstance of our formulated model, investigation was initiated for situation where infected patients had no access to medicated treatment but were rather left to the critical role of adaptive natural immune effectors response. Results showed that for a patient with harmonized immune effectors, quantifiable healthy $\mathrm{CD}^{+} \mathrm{T}$ cells are sustained with flash peak value of $U_{T}(t) \leq 23.641 \mathrm{cell} \mu l^{-1}$ at the interval $14 \leq t_{f} \leq 16$ months. On the other hand, infected $\mathrm{CD}^{+} \mathrm{T}$ cells reduced to near zero in the same time interval but thereafter submerges. The toxicity of both virions exhibited slight sustainability at the early time interval of $t_{f} \leq 10$ months and thereafter declined. The critical role of both CTLp and CTLe is evidence by the ever positive values with slight decline due to possible natural clearance rate. This situation affirmed the minimal sustainability of healthy $\mathrm{CD} 4^{+} \mathrm{T}$ cells by infected patient. Moreso, increase sustenance of virions aggressiveness ascertains the absence of chemotherapy at this situation.

Further deduction following the introduction of treatment functions resulted to enhanced maximization of healthy $\mathrm{CD}^{+} \mathrm{T}$ cells with steady increase to $U_{T}(t) \leq 9.328 \mathrm{cell} \mu \mathrm{l}^{-1}$ while infected $\mathrm{CD}^{+} \mathrm{T}$ cells were eliminated after $t_{f} \leq 16$ months. This situation was visualized with the elimination of both virions at the $16^{\text {th }}$ month of active chemotherapy application. The model thus aligned the study by [18], which had set upperbounds time benefit on cost to be 480 days $\cong 16$ months. This outcome suggests possible termination of chemotherapy administration and concurrently accounted for any possible drug side-effects. Furthermore, the ascertainment of the present investigation is ascertained by the complete elimination of intrinsic virulence of infectious viruses as in fig. 3(d). The implication is that with the application of chemotherapy treatments, linear growth of intrinsic virulence is reversed. Moreso, we as well observed that prolongation of chemotherapies, which boosted CTLs leads to significant maximization of healthy $\mathrm{CD}^{+} \mathrm{T}$ cells and are in collaboration with the experimental findings of $[14,29,30]$.

Thus, the overall results which indicated $t_{f} \leq 16$ months of cohesive treatment administration, translate to the desired minimization of cost of medication. Moreso, the decline in infected $\mathrm{CD} 4^{+} \mathrm{T}$ cells following the presence of treatment functions were comprehensively vindicated by the sharp decline of dual virions to very low level with healthy $\mathrm{CD} 4^{+} \mathrm{T}$ cells sustained at maximum level and linear expansion of both precursors and effectors of CTLs.

\section{CONClusion}

In overcoming the weakness identified from a number of related scientific investigations, this paper had formulated using ODEs, a novel 7-Dimensional non-linear mathematical dynamic model for the optimal control treatment of asymptomatic dual HIV-pathogen infections on host target $\mathrm{CD} 4{ }^{+} \mathrm{T}$ cells. The study adopted quadrupled treatment functions with model constructed as a time optimal control problem and analyzed using linear programing approximation method. Results of numerical simulations affirmed the maximization of healthy $\mathrm{CD}^{+}{ }^{+}$T-lymphocytes, suppression of dual HIV- 
Quantitative Approximability of Optimal Control by Linear Programing Model for Asymptomatic Dual HIV - Pathogen Infections

pathogen infectivity, increase sustainability of dual CTLs critical role, early elimination of virions aggressiveness with an overall minimization of benefit on cost. Therefore, the result which justified the application of linear programing approximability approach, strongly advocates the inclusion of delay intracellular component on state variables as an enhancement in future studies.

\section{ACKNOWLEDGMENTS}

My amiable appreciation goes to theEditor-in-Chief of IJSIMR-Dr. K.V.L.N.Achrayulufor the outstanding academic relationship and the high research standard set for this journal. Also, I sincerely appreciate the valuable comments from my anonymous referee(s) for his/her, which have led to an improved version of this manuscript.

\section{REFERENCES}

[1] Craig, I. and Xia, X. (2005) Can HIV/AIDS be Control? IEEE Control Syst, Mag., 25(1), 80-83.

[2] Di-Masco, M. (2004) Modeling the Long-term Control of Viremia in HIV-1 Infected Patients Treated with Antiretroviral Therapy. Math, Biosci., 188, 47-62.

[3] Perelson, A. S., Neumann, A. U., Markowitz, M., Leonard, J. M. and Ho, D. D. (1996) HIV-1 Dynamics in-Vivo-virion Clearance Rate, Infected Cell Life-span, and Viral Generation Time. Science, 271(5255): 1582-1586.

[4] Kirschner, D. E. and Perelson, A. S. (1995) A model for the Immune System Response to HIV: AZT Treatment Studies. In: Arino, O., Axelrod, D., Kimmel, M., Langlais, M. (eds), Mathematical Population Dynamics: Analysis of Heterogeneity, Vol. 1: Theory of Epidemics, Wuerz Pub. Ltd.,Winnipeg, Canada, 295-310.

[5] Kirschner, D., Lenhart, S. and Serbin, S. (1997) Optimal Control of the Chemotherapy of HIV. Journal of Mathematical Biology, 35, 775-792.

[6] Kirschner, D. and Webb, G. F. (1996) A Model for Treatment Strategy in the Chemotherapy of AIDS.Bull. Math. Biol., 58: 367-390

[7] Wein, L. M., Zenios, S. A. and Nowak, M. A. (1997) Dynamic Multidrug Therapies for HIV: A control Theoretic Approach. J. Theor. Biol,.185, 15-29.

[8] Xia, X. (2003) Estimation of HIV/AIDS Parameters. Automatica, 39, 11, 1983-1988.

[9] Bassey, E. B. and Lebedev, K. A. (2016) On Analysis of Parameter Estimation Model for the Treatment of Pathogen-Induced HIV Infectivity. Open Access Library Journal, 3(4): 1-13

[10] Butler S., Kirschner, D. and Lenhart S. (1997) Optimal Control of the Chemotherapy Affecting the Infectivity of HIV, Editors: O. Arino, D. Axelrod and M. Kimmel. Advances in Mathematical Population Dynamics- Molecules, Cells and Man, 6, 557-569.

[11] Adams, B. M., Banks, H.T., Hee-Dae K. and Tran, H.T. (2004) Dynamic Multidrug Therapies for HIV: Optimal and STI Control Approaches. http://citeseerx.ist.psu.edu/viewdoc/summary?doi=10.1.1.400.9056 .Accessed October 05, 2017.

[12] Zhu, H. and Zou, X. (2008) Impact of Delay in Cell Infection and Virus Production on HIV-1 Dynamics. Math.Medic.Bio., 25, 99-112.

[13] Adams, B. M., Banks, H. T., Davidian, M., Kwon, Hee-Dae, Tran, H. T., Wynne, S. N. and Rosenberg, E.S. (2005) HIV Dynamics: Modeling, Data Analysis, and Optimal Treatment Protocols. J. Comp. Appl. Math., 184, 10-49. http://citeseer.ist.psu.edu/viewdoc/summary?doi=10.1.1.115.8107 . Accessed October 05, 2017.

[14] in, X., et al., (2000) An Antigenic Threshold for Maintaining Human Immunodeficiency Virus Type 1Specific Cytotoxic T-lymphocytes. Mol. Med., 6, 803-809.

[15] Hattaf, K. and Yousfi, N. (2012) Optimal Control of a Delayed HIV Infection Model with Immune Response Using an Efficient Numerical Method. Biomathematics, 2012, 1-7.

[16] Bassey, E. B. (2017) Optimal Control Model for Pair Chemotherapy Treatment with Time-delay Immunity in Dual HIV-Infectivity. Applied Mathematics and Physics, 5, 2, 61-76.

[17] Wodarz, D. and Nowak, M.A. (2002) Mathematical Models of HIV Pathogenesis and Treatment. BioEssays: Wiley periodicals, Inc., 24, 1178-1187.

[18] Zarei, H., Kamyad, A. V. and Effati, S. (2010) Maximizing of Asymptomatic Stage of Fast Progressive HIV Infected Patient Using Embedding Method. Intelligent Control and Automation, 1: 48-58.

[19] Culshaw, R., Ruan, S. and Spiteri, R. J. (2004) Optimal HIV Treatment by Maximizing Immune Response. Journal of Mathematical Biology, 48, 5, 545-562. 
Quantitative Approximability of Optimal Control by Linear Programing Model for Asymptomatic Dual HIV - Pathogen Infections

[20] Bassey, E. B., Kimbir, R. A. and Lebedev, K. A. (2016) On Optimal Control Model for the Treatment of Dual HIV-Parasitoid Pathogen Infection.J Bioengineer \& Biomedical Sci., 7: 212, 1-7. doi: 10.4172/21559538.1000212.

[21] Landi, A., Mazzoldi, A., Andreoni, C., Bianchi, M., Cavallini, A., Laurino, M., Ricotti, L., Iuliano, R., Matteoli, B. and Ceccherini-Nelli, L. (2008) Modelling and Control of HIV Dynamics. Computer Methods and Programs in Biomedicine, 89, 2, 162-168.

[22] Hale, J. and Verduyn Lunel, S. M. (1993) Introduction to Functional Differential Equations. Applied Mathematical Science, 99, Springer-Verlag, New York.

[23] Zhu, H. and Zou, X. (2009) Dynamics of a HIV-1 Infection Model with Cell-Mediated Immune Response and Intracellular Delay. Discrete and continuous dynamical systems, series B, 12, 2, 511-524.

[24] Young, C. (1969) Calculus of Variations and Optimal Control Theory. Sunders, Philadelphia, USA.

[25] Wilson, D. A. and Rubio, J. E. (1977) Existence of Optimal Controls for the Diffusion Equation. Journal of OptimizationTheory and Applications, 22, 91-101.

[26] Rubio, J. E. (1986) Control and Optimization: The Linear Treatment of Non-linear Problems. Manchester UniversityPress, Manchester.

[27] Mehne, H. H., Farahi, M. H. and Kamyad, A. V. (2005) MILP Modelling for the Time Optimal Control Problem in The Case of Multiple Targets. Optimal Control Applications and Methods, 27, 2, 77-91.

[28] Bassey, B. E. (2017) Quantum Optimal Control Dynamics for Delay Intracellular and Multiple Chemotherapy Treatment (MCT) of Dual Delayed HIV - Pathogen Infections . International Journal of Scientific and Innovative Mathematical Research, vol. 5, no. 6, p. 1-19,http://dx.doi.org/10.20431/23473142.0506001

[29] Bassey, E. B. (2017) Dynamic optimal control model for periodic multiple chemotherapy (PMC) treatment of dual HIV - pathogen infections. J Anal Pharm Res 6(3): 00176, 1-22. DOI: 10.15406/japlr. 2017.06.00176

\section{AUTHOR'S BIOGRAPHY}

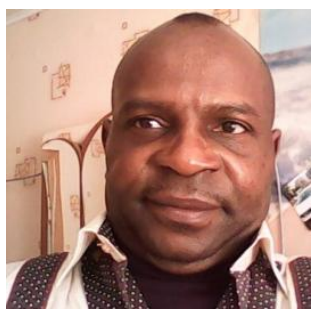

Bassey Echeng Bassey, a $\mathrm{PhD}$ holder in Computational Mathematics and Informatics from Kuban State University, Krasnodar Russia and M.Sc. in Industrial Mathematics, Federal University of Agriculture, Makurdi, Nigeria. $\mathrm{He}$ is a lecturer and presently the Co-coordinator - Head of Pre-degree program, Cross River University of Technology, Calabar, Nigeria. Published more than 23 papers in reputed journals, monographs' and authorof textbooks. Research interests include but not limited to: Mathematical modeling, numerical methods; simulation analysis, mathematical methods, computer technology and programming.

Citation: B. E. Bassey, "Quantitative Approximability of Optimal Control by Linear Programing Model for Asymptomatic Dual HIV - Pathogen Infections ", International Journal of Scientific and Innovative Mathematical Research, vol. 5, no. 9, p. 01-21, 2017., http://dx.doi.org/10.20431/2347-3142.0509001

Copyright: () 2017 Authors. This is an open-access article distributed under the terms of the Creative Commons Attribution License, which permits unrestricted use, distribution, and reproduction in any medium, provided the original author and source are credited. 